\section{Proximate determinants}

\section{of telomere length in sand lizards (Lacerta agilis)}

\author{
Mats Olsson ${ }^{1,2, *}$, Angela Pauliny ${ }^{2}$, Erik Wapstra ${ }^{3}$ \\ and Donald Blomqvist ${ }^{2}$ \\ ${ }^{1}$ School of Biological Sciences, University of Wollongong, Wollongong, \\ New South Wales 2522, Australia \\ ${ }^{2}$ Department of Zoology, University of Gothenburg, PO Box 463, \\ 40530 Gothenburg, Sweden \\ ${ }^{3}$ School of Zoology, University of Tasmania, Hobart, \\ 7001 Tasmania, Australia \\ *Author for correspondence (molsson@uow.edu.au).
}

Telomeres are repeat sequences of non-coding DNA that cap the ends of chromosomes and contribute to their stability and the genomic integrity of cells. In evolutionary ecology, the main research target regarding these genomic structures has been their role in ageing and as a potential index of age. However, research on humans shows that a number of traits contribute to among-individual differences in telomere length, in particular traits enhancing cell division and genetic erosion, such as levels of free radicals and stress. In lizards, tail loss owing to predation attempts results in a stress-induced shift to a more cryptic lifestyle. In sand lizard (Lacerta agilis) males, telomere length was compromised by tail regrowth in a body size-related manner, so that small males, which already exhibit more cryptic mating tactics, were less affected than larger males. Tail regrowth just fell short of having a significant relationship with telomere length in females, and so did age in males. In females, there was a significant positive relationship between age and telomere length. We conclude that the proximate effect of compromised antipredation and its associated stress seems to have a more pronounced effect in males than in females and that age-associated telomere dynamics differ between the sexes.

Keywords: autotomy; telomere length; predation stress

\section{INTRODUCTION}

Telomeres are currently thought to have several vital functions in addition to protection of the chromosome ends, such as providing a mechanism for distinguishing between real chromosome ends and breaks that need repairing, a role in alignment and segregation of chromosomes during meiosis, modifying gene expression, and contributing to stress resistance in cells and tissue (Baur et al. 2001; Blackburn 2005; Monaghan \& Haussman 2006; Kappei \& LondoñoVallejo 2008). Dysfunctional telomeres render the genome of the cell unstable, since the uncapped chromosome can no longer be distinguished from double-stranded chromosome breaks, which evokes cellular repair mechanisms (Monaghan \& Haussman 2006). This leads to apoptosis (cell death) or replicative senescence, that is, the cell stops dividing. When telomeres become critically short, the resulting dysfunction contributes to risk of disease and reduced lifespan. For instance, telomere shortening is linked to age-associated risks of mortality from infection and heart disease (Kappei \& Londoño-Vallejo 2008), and may be the effect of genetic erosion associated with increased metabolic rate or free radical attack (Von Zglinicki 2002), or other forms of stress (Epel et al. 2004). This is an overlooked area, since not only will such effects influence direct links between age and telomere length, but they may also reveal proximate effects and associated fitness costs of telomere shortening from a variety of stressors.

One of the most stressful events in an animal's life is exposure to an overt predation attempt. In some lizards, a well-known easy way out of this lifethreatening dilemma is to 'drop' ones tail (Ballinger 1973; Dial \& Fitzpatrick 1981; Cooper \& Wilson 2008; Cooper \& Frederick 2010). However, a large body of literature has confirmed that tail autotomy and the associated regeneration of the tail leads to associated costs, such as visibility, reduced sprint speed, growth rate and clutch size (Cooper \& Wilson 2008; Cooper \& Frederick 2010). In sand lizards (Lacerta agilis), tail regeneration results in a new tail with cartilage as support tissue, but no formation of new vertebrae with fracture planes that can be 'reused' for antipredation behaviour. Thus, once a tail is completely lost, there is no way to lose the tail 'voluntarily' under predator attack, which itself is likely to be highly stressful and seriously compromise survivorship (Ballinger 1973; Dial \& Fitzpatrick 1981; Cooper \& Wilson 2008; Cooper \& Frederick 2010). Growth is continuous and only asymptotically approaching zero in this species (Olsson \& Shine 1996). In the current study, we therefore use the length of the regrown tail $(\mathrm{mm})$ as a proxy for exposure time to predators under compromised antipredation behaviour.

\section{MATERIAL AND METHODS}

Sand lizards (L. agilis) are small (to $20 \mathrm{~g}$ ) ground-dwelling lacertid lizards. Males have a green area on their body sides that increases proportionally with size and age, so that small males have a much more cryptic reproductive tactic than large dominant males (Olsson 1994a). Females are cryptically coloured and have a sedentary lifestyle (Olsson 1994a). Our free-ranging population (Asketunnan, Sweden approx. $57^{\circ} 22^{\prime} \mathrm{N}, 11^{\circ} 58^{\prime} \mathrm{E}$ ) has been studied for 25 years and detailed field protocols have been published elsewhere (Olsson 1994a; Olsson \& Shine 1996; Olsson et al. 1996). In brief, individually marked lizards were monitored in the wild after measuring, weighing, ectoparasite counting, photography of male body sides and blood sampling as per standard protocols (Olsson 1994a; Olsson \& Shine 1996; Olsson et al. 1996). A male's green lateral area (the 'badge') was measured as the ratio of area of green to total area using photographs (Olsson 1994a; Olsson \& Shine 1996; Olsson et al. 1996). Age in years was calculated from known hatching records (Olsson 1994a; Olsson \& Shine 1996; Olsson et al. 1996). We included the same variables for analyses in both sexes (snout-vent length $(\mathrm{mm})$, tail regeneration $(\mathrm{mm})$ and their interaction to look for size-dependent effects (expected particularly in males), number of observations as a proxy for exposure, number of ectoparasites as a proxy of health (Uller et al. 2003) and age (years)). In males, we also included the sexlimited badge size, since this has been shown to be correlated with a number of fitness components in males (Olsson et al. 2000). Predictors were backward-eliminated at $p>0.25$ until a last model when $p>0.30$ was accepted (because 'badge' and 'ticks' were border-line cases and kept in this model).

DNA extractions followed a standard protocol (Pauliny et al. 2006). Telomere restriction fragments (TRFs) were prepared as 
described previously (Pauliny et al. 2006). After standard Southern blotting, TRFs were hybridized to an alkaline phosphatase-linked telomere probe and detected by chemoluminescence (AlkPhos labelling and detection kit, GE Healthcare). Digitalized signals were analysed as described previously (Pauliny et al. 2006) within a window of approximately $9-30 \mathrm{~kb}$ to avoid signals from telomericlike interstitial regions (Delany et al. 2000). The analysis of TRFs with TELOMETRIC complied with all conditions for which the program has been validated (Grant et al. 2001; M. Ochs 2009, personal communication).

\section{RESULTS}

In females, snout-vent length and number of observations were non-significant predictors of telomere length $(p>0.30)$, and ticks (means \pm s.d. given for all traits, $12.0 \pm 7.7$ s.d.) and tail regeneration (30.3 mm \pm 27.2 s.d.) fell just short of significance (table 1). Age, however, was correlated with telomere length, but in a positive way (table 1; range 2-8 years, TRF: range $17.0-20.2 \mathrm{~kb}$ ). Since this could be either because of females with longer telomeres surviving longer or because telomeres increase in telomere length with age, we double-checked the length of females for which we had repeat samples at different ages. For nine females, we had two samples from different years, and in these individuals, there was no significant change in mean TRF with age (time between sampling 1-4 years, mean difference in $\mathrm{TRF}=-0.196 \pm 0.26$ s.e.; pairwise $t$-test, $t=-0.75, p=0.47, n=9)$. Furthermore, female growth rate (residuals from a snout-vent length-age regression) was uncorrelated with tail regrowth (residuals from a regrowth-mass regression; $r=0.13$, $p=0.45, n=32$ ).

Males exhibited a very different scenario. The number of times a male was observed was a significant predictor of telomere length (means \pm s.d. given for all traits, $4.7 \pm 2.8$ s.d.), age fell short of significance ( 3.8 years, \pm 1.1 s.d. $)$ and badge size $(61 \pm 12 \%$, s.d. $)$ and tick load $(25.7 \pm 16.1$ s.d.) were both non-significant predictors (table 1). However, tail regeneration (30.9 mm, \pm 26.6 s.d.) and snout-vent length $(73.3 \mathrm{~mm}, 4.2 \mathrm{s.d}$.) were both significant predictors as was their interaction effect (table 1). In order to assess what drives this interaction, we split the male sample at the mean snout-vent length $(73.3 \mathrm{~mm})$ into two size classes and ran a homogeneity of slopes test to assess whether one size class had a steeper slope between regeneration length and telomere length with the other variables in table 1 as covariates. The interaction between male size class and regeneration was indeed significant $(\beta=0.013, \pm 0.01, t=$ 2.08, $F_{1,74}=4.3, p=0.04$; the regression coefficient for large males is given as a contrast to a slope set to zero for small males in Proc GLM, SAS, and a generalized inverse was used to solve the normal equation). Furthermore, for males, there was a significant trade-off between growth rate $\left(\mathrm{mm} \mathrm{yr}^{-1}\right)$ and tail regrowth (mm tail $/ \mathrm{mm}$ snout-vent length; $r=-0.28, p=0.012, n=81)$.

\section{DISCUSSION}

Recent research has shown that telomere dynamics are substantially more complex than 'age-related
Table 1. Multiple regression analyses of modulators of telomere length in male and female sand lizards. s.e., standard error. $\beta$, regression coefficient. d.f. for all traits is 1 with the error given by the global models. SVL, snout-vent length (mm); age (years); observations (counts); ticks (counts); regeneration $(\mathrm{mm}) .{ }^{*} p<0.05 ; * * * p<0.001$.

\begin{tabular}{lcccl} 
males (global model: & $\left.F_{7,68}=4.13, p=0.0008, R^{2}=0.30\right)$ \\
\hline predictors & $\beta$ & s.e. & $t$ & $p$ \\
\hline age & -0.12 & 0.07 & -1.82 & 0.074 \\
observations & 0.10 & 0.03 & 3.52 & $0.0008^{* * *}$ \\
badge & 0.87 & 0.74 & 1.17 & 0.25 \\
ticks & -0.00 & 0 & -1.07 & 0.29 \\
regeneration & -0.13 & 0.05 & -2.55 & $0.013^{*}$ \\
snout-vent length & -0.06 & 0.02 & -2.44 & $0.017^{*}$ \\
SVL $\times$ regeneration & 0.002 & 0 & 2.51 & $0.0143^{*}$ \\
\hline females (global model: & $\left.F_{3,56}=4.64, p=0.006, R^{2}=0.20\right)$ \\
ticks & 0.02 & 0.01 & 1.76 & 0.08 \\
age & 0.14 & 0.06 & 2.36 & $0.022^{*}$ \\
regeneration & -0.005 & 0 & -1.68 & 0.10 \\
\hline
\end{tabular}

shortening', accepted as a dogma for substantial time (Monaghan \& Haussman 2006), and its telomeraseinduced maintenance in many tissue and taxa (Kappei \& Londoño-Vallejo 2008). A logical and much more pleasing scenario for an evolutionary biologist is to view telomere length as a result of opposing pressures of selection with pros (sustained genomic stability) and cons (e.g. risk of cancer) moulding this process. In this framework, it is also much more straightforward to understand, and predict, how sexspecific effects may evolve, how the rate of telomere reconstruction may change with age and associated metabolic rate shifts and how clade-specific telomere characteristics arise in phylogenies (Kappei \& Londoño-Vallejo 2008).

In the current study, we assess in particular how a vital aspect of antipredation-tail autotomy-is linked to telomere length in two sexes that differ substantially in behaviour. Female sand lizards are characteristically cryptically coloured, live a sedentary life with a home range about a tenth the area of males (Olsson et al. 1996) and with a regrowth of tail not significantly traded-off by growth rate. This seems to suggest a life under relatively little stress from predation compared with males (but see Olsson $1994 b)$. In contrast, male tail regeneration is compromised by a high growth rate, perhaps because of selection for rapid growth in males that have more size-constrained reproductive success (Olsson \& Shine 1996), and large males have a highly conspicuous phenotype and a reproductive success constrained by mate acquisition and mobility (Olsson et al. 2000). Thus, males 'in-the-fast-lane' would be predicted to become more stressed during the mating season-and that is exactly what we see (Olsson et al. 2005). Males that are engaged in more contests for partners have higher corticosterone levels (Olsson et al. 2005), and these are exactly the same males in which tail regrowth most strongly seems to compromise telomere length. 
Our results show that telomere shortening not only 'ticks-away' in some pre-determined pattern set by prior selection but can also be moderated in free-ranging populations by processes set by sexually selected life-history tactics that show sexually dimorphic patterns, as predicted by evolutionary theory. This encourages sex-specific analysis of telomere dynamics in the wild. We are grateful to the Swedish Science Council and the Australian Research Council for financial support (M.O.).

Ballinger, R. E. 1973 Experimental evidence of the tail as a balancing organ in the lizard Anolis carolinensis. Herpetologica 29, 65-66.

Baur, J. A., Zou, W., Shay, J. W. \& Wright, W. E. 2001 Telomere position effect in human cells. Science 292, 2075-2077. (doi:10.1126/science.1062329)

Blackburn, E. H. 2005 Telomeres and telomerase: their mechanisms of action and the effects of altering their functions. FEBS Lett. 579, 859-862. (doi:10.1016/ j.febslet.2004.11.036)

Cooper, W. E. \& Frederick, W. G. 2010 Predator lethality, optimal escape behavior, and autotomy. Behav. Ecol. 21, 91-96. (doi:10.1093/beheco/arp151)

Cooper, W. E. \& Wilson, D. S. 2008 How to stay alive after losing your tail. Behaviour 145, 1085-1099. (doi:10. $1163 / 156853908784474515)$

Delany, M. E., Krupkin, A. B. \& Miller, M. M. 2000 Organization of telomere sequences in birds: evidence for arrays of extreme length and for in vivo shortening. Cytogenet. Cell. Genet. 90, 139-145.

Dial, B. E. \& Fitzpatrick, L. C. 1981 The energetic costs of tail autotomy to reproduction in the lizard Coleonyx brevis (Sauria: Gekkonidae). Oecologia 51, 310-317. (doi:10. 1007/BF00540899)

Epel, E. S., Blackburn, E. H., Lin, J., Dhabhar, F. S., Adler, N. E., Morrow, J. D. \& Cawthon, R. M. 2004 Accelerated telomere shortening in response to life stress. Proc. Natl Acad. Sci. USA 101, $17312-17$ 315. (doi:10.1073/pnas. 0407162101)

Grant, J. D., Broccoli, D., Muquit, M., Manion, F. J., Tisdall, J. \& Ochs, M. F. 2001 Telometric: a tool providing simplified, reproducible measurements of telomeric DNA from constant field agarose gels. Biotechniques 31, 1314-1318.

Kappei, D. \& Londoño-Vallejo, J. A. 2008 Telomere length inheritance and aging. Mech. Ageing Dev. 129, 17-26. (doi:10.1016/j.mad.2007.10.009)

Monaghan, P. \& Haussman, M. F. 2006 Do telomere dynamics link lifestyle and lifespan? Trends Ecol. Evol. 21, 47-53. (doi:10.1016/j.tree.2005.11.007)

Olsson, M. 1994a Nuptial coloration in the sand lizard, Lacerta agilis: an intrasexually selected cue to fighting ability. Anim. Behav. 48, 607-613. (doi:10.1006/anbe. 1994.1280)

Olsson, M. $1994 b$ Nuptial coloration and predation risk in model sand lizards, Lacerta agilis. Anim. Behav. 46, 410-412. (doi:10.1006/anbe.1993.1207)

Olsson, M. \& Shine, R. 1996 Does reproductive success increase with age or with size in a species with indeterminate growth? - a case study using sand lizards Lacerta agilis. Oecologia 105, 175-178.

Olsson, M., Gullberg, A. \& Tegelström, H. 1996 Malformed offspring, sibling matings, and selection against inbreeding in the sand lizard (Lacerta agilis). F. Evol. Biol. 9, 229-242. (doi:10.1046/j.1420-9101.1996. 9020229.x)

Olsson, M., Wapstra, E., Madsen, T. \& Silverin, B. 2000 Testosterone, ticks, and travels: a test of the immunocompetence-handicap hypothesis in free-ranging male sand lizards. Proc. R. Soc. Lond. B 267, 2339-2343. (doi:10. $1098 / \mathrm{rspb} .2000 .1289$ )

Olsson, M., Madsen, T., Wapstra, E., Silverin, B., Ujvari, B. \& Wittzell, H. 2005 MHC, health, color, and reproductive success in sand lizards. Behav. Ecol. Sociobiol. 58, 289-294. (doi:10.1007/s00265-005-0921-y)

Pauliny, A., Wagner, R. H., Augustin, J., Szép, T. \& Blomqvist, D. 2006 Age-independent telomere length predicts fitness in two bird species. Mol. Ecol. 15, 1681-1687. (doi:10.1111/j.1365-294X.2006.02862.x)

Uller, T., Olsson, M. \& Madsen, T. 2003 Family and population effects on disease resistance in a reptile. Heredity 91, 112-116. (doi:10.1038/sj.hdy.6800288)

Von Zglinicki, T. 2002 Oxidative stress shortens telomeres. Trends Biochem. Sci. 27, 339-344. (doi:10.1016/S09680004(02)02110-2) 